\title{
Análisis psicométrico de la Escala de Satisfacción Familiar de Wilson y Olson en una muestra de trabajadores de Arequipa
}

\section{PSYCHOMETRIC ANALYSIS OF WILSON AND OLSON'S FAMILY SATISFACTION SCALE IN A SAMPLE OF WORKERS FROM AREQUIPA}

\author{
Walter L. Arias Gallegos'1, Renzo Rivera Calcina'1, Karla D. Ceballos Canaza' \\ 1. Universidad Católica San Pablo, Arequipa, Perú.
}

\section{RESUMEN}

En este trabajo se realizó un análisis psicométrico de la Escala de Satisfacción Familiar de Olson y Wilson en una muestra de 274 trabajadores de la ciudad de Arequipa, con la finalidad de valorar su estructura factorial y ofrecer un instrumento válido y confiable para evaluar la funcionalidad familiar en contextos laborales. Los resultados indican que la dimensión de Cohesión cuenta con un indice de confiabilidad de $\alpha=0,858$ y la dimensión de Adaptabilidad de $\alpha=0,848$. Asimismo el análisis factorial confirmatorio revela indicadores de bondad de ajuste adecuados, que confirman la estructura bifactorial de la prueba. También se ofrecen baremos con percentiles en tres niveles (bajo, moderado y alto) de la funcionalidad familiar, que permitirán valorar el sistema familiar como factor de riesgo psicosocial en el trabajo.

(Arias W, Renzo Calcina R, Ceballos K, 2018. Análisis psicométrico de la Escala de Satisfacción Familiar de Wilson y Olson en una muestra de trabajadores de Arequipa. Cienc Trab. Ene-Abr; 20 [61]: 56-60).

Palabras clave: SATISFACCIÓN FAMILIAR, PSICOMETRÍA, ANÁLISIS FACTORIAL CONFIRMATORIO, MODELO CIRCUNFLEJO.

\section{ABSTRACT}

In this work we made a psychometric analysis about Olson and Wilson's Family Satisfaction Scale in a sample of 274 workers from Arequipa City, in order to value its factor structure and offer a valid and reliable instrument to test the family functionability in organizational contexts. The results indicate that the dimension of Cohesion has an index of reliability of $\alpha=0.858$, and the dimension of Adaptability has a reliability index of $\alpha=0.848$. Moreover, the confirmatory factor analysis reveal indicators of adequate good fitness index, which confirm the bifactorial structure of the test. Finally, we show percentiles in three levels, about the family functionability, so they could allow the evaluation of family as a factor of psychosocial risk at work.

Key words: FAMILY SATISFACTION, PSYCHOMETRICS, CONFIRMATORY FACTORIAL ANALYSIS, CIRCUMPLEX MODEL.

\section{INTRODUCCIÓN}

El tema de la familia ha cobrado cada vez mayor relevancia en el mundo laboral, debido primordialmente a los cambios en la estructura familiar que se han venido presentando, especialmente en el siglo XX. Tal es así que las guerras mundiales, la creciente tasa de divorcios y el acceso de la mujer a la educación superior han impulsado su inserción en el mundo laboral, con implicancias muy importantes para la organización del trabajo. Por ello, hoy en día se habla del conflicto trabajo-familia o familia-trabajo ${ }^{1}$ que comprende la interdependencia entre la vida familiar y las funciones laborales, de modo que la primera puede ser obstáculo para las segundas, o vice-

Correspondencia / Correspondence:

Walter L. Arias Gallegos

Universidad Católica San Pablo

rb. Campiña Paisajista s/n Quinta Vivanco,

Cercado. Arequipa, Perú.

Tel.: (51-54) 608020.

e-mail: walterlizandro@hotmail.com.

Recibido: 30 de Octubre de 2017 / Aceptado: 05 de Enero de 2018.

versa. En ese sentido, se ha reportado que los varones perciben que la familia interfiere con su trabajo, mientras que para las mujeres el trabajo interfiere con sus responsabilidades familiares. ${ }^{2}$

En torno a este tema se han considerado diversos aspectos de tipo sociocultural sobre los roles de género, que favorecen a los varones y perjudican a las mujeres, pues son ellas quienes tienen un doble rol más acentuado como mujeres trabajadoras y como madres de familia. ${ }^{3}$ Aunque actualmente los varones asumen cada vez más funciones familiares, en la mayoría de los casos la carga familiar es más pesada para las mujeres.

Este panorama supone hacer estudios sobre la ecuación familiatrabajo que nos permitan comprender y actuar sobre una realidad compleja que afecta a la familia y sus miembros, las instituciones laborales y la sociedad en su conjunto. Lamentablemente, en América Latina existen pocos estudios sobre esta problemática, pero algunos estudios señalan que existe un conflicto trabajo-familia moderado y que este se relaciona inversamente con el bienestar psicológico de los trabajadores chilenos. ${ }^{4}$ Para reducir este conflicto y propender hacia un balance entre el trabajo y la familia, en Colombia se han propuesto horarios flexibles, diversas modalidades de teletrabajo, políticas de promoción de la familia y otras alternativas que buscan mitigar los efectos de la sobrecarga laboral en la 
familia, o armonizar los estilos de vida en la familia con las metas organizacionales. ${ }^{5}$

Otras aproximaciones, han intentado medir el conflicto trabajofamilia a través del diseño y validación de instrumentos, como es el caso de Venezuela, donde Cachutt y Ortiz han planteado una serie de indicadores de la integración trabajo-familia. ${ }^{6}$ En el caso del Perú, existe una diversidad de estudios relativos a la familia y su impacto en la educación de los hijos y la salud física y mental de sus miembros $^{7}$, así como a diversos aspectos organizacionales en materia de seguridad y riesgo psicosocial o del capital social y los recursos humanos $^{8}$, pero no existen estudios sobre las relaciones entre la familia y el trabajo.

Una excepción podría ser el estudio de Prado y del Águila9, que valoran el grado de satisfacción y ajuste marital en parejas que trabajan en la ciudad de Lima. Por otro lado, en Arequipa (segunda ciudad del Perú, ubicada al sur del país), recientemente se ha publicado un estudio en el que se reporta que la integración familiar tiene un efecto amortiguador en la satisfacción laboral cuando el trabajador presenta altos niveles de estrés. ${ }^{10}$ En ese sentido, en Arequipa -como en el Perú- la investigación de la familia ha sido recurrente, de modo que se pueden encontrar estudios sobre el efecto de la estructura familiar en variables socioemocionales de los hijos ${ }^{11,12}$ y el aprovechamiento escolar ${ }^{13}$, la violencia de pareja ${ }^{14}$, diferencias entre casados y divorciados ${ }^{15,16,17}$, la felicidad y la integración familiar ${ }^{18}$, etc. Con respecto al trabajo y la familia, se han publicado estudios sobre la situación laboral y familiar de las mujeres que ocupan cargos directivos ${ }^{19}$, el impacto de la estructura familiar en la economía y el trabajo $^{20,21}$ y la influencia de diversas variables sociolaborales en la integración familiar. ${ }^{22}$ Ninguno de estos trabajos, empero, aborda el tema desde la perspectiva del conflicto familia-trabajo, salvo el mencionado estudio. ${ }^{10}$

En ese sentido, un factor que dificulta la investigación de este tema es la falta de instrumentos validados en la población peruana o en muestras locales. Aunque sí es posible encontrar instrumentos estandarizados que evalúan el funcionamiento familiar en el Perú, la gran mayoría se ha validado en muestras de adolescentes o estudiantes universitarios ${ }^{23,24,25,26}$ y no en trabajadores, como sí ocurre en otros países de Iberoamérica. ${ }^{6,27}$ El objetivo de este estudio es presentar un análisis psicométrico de la Escala de Satisfacción Familiar de Olson y Wilson en una muestra de trabajadores de la ciudad de Arequipa, con el fin de valorar su validez, confiabilidad y verificar su estructura factorial.

Esta prueba fue construida inicialmente por David Olson en 1985 en base a dos dimensiones, como son la cohesión familiar y la adaptabilidad desde un enfoque sistémico ${ }^{28}$, conocido como modelo circunflejo de la familia que, según el grado de caracterización de los sistemas familiares en función de los límites y roles, su flexibilidad, reglas, estilos de negociación y convivencia determinan su funcionalidad familiar. ${ }^{29}$ Sin embargo, la Escala de Satisfacción Familiar cuenta con diversas versiones, unas extensas o abreviadas y otras con diversos formatos de respuesta. Por ejemplo, desde 1985 hasta el 2003, se tienen cuatro versiones de las Escalas de Cohesión y Adaptabilidad de Olson, conocido como FACES. De hecho, para la tercera edición, Olson agregó una tercera dimensión, que denominó Comunicación $^{30}$, y que al ser revisada en países como Japón, Chile, México y Argentina ha reportado resultados contradictorios. ${ }^{31}$

Para el caso de Perú, Rosa María Reusche fue la primera autora en trabajar con el modelo circunflejo y la Escala de Satisfacción Familiar en $1994 .{ }^{32}$ Luego, varios estudios han acogido el modelo circunflejo para investigar la funcionalidad familiar con respecto a la salud mental de los hijos adolescentes y jóvenes universitarios. ${ }^{24-26,33}$ Recientemente, se publicó un estudio realizado en Lima con 910 estudiantes adolescentes entre 11 y 18 años, con la finalidad de valorar la validez y la confiabilidad del instrumento, confirmando la estructura factorial de la prueba según el modelo de Olson. ${ }^{26}$ De nuestra parte, se pretende analizar las propiedades psicométricas de la Escala de Satisfacción Familiar de Olson y Wilson, que cuenta con dos dimensiones solamente -cohesión y adaptabilidad- en una muestra de trabajadores de la ciudad de Arequipa. La finalidad de este estudio es ofrecer un instrumento de investigación familiar validado que permita evaluar la satisfacción familiar de los trabajadores y así poder contar con herramientas útiles para la valoración del conflicto trabajo-familia. En el siguiente apartado se presentan los criterios metodológicos que se emplearon para cumplir con nuestro objetivo.

\section{MÉTODO}

El presente estudio es de tipo instrumental ${ }^{34}$, pues se pretende valorar las propiedades psicométricas de un instrumento para la valoración de la funcionalidad familiar.

\section{Muestra}

Está conformada por una muestra multiocupacional de 274 trabajadores de la ciudad de Arequipa. La edad promedio fue de 42 años con una desviación estándar de \pm 9,4 dentro de un rango de 22 a 70 años. El 59,4\% son mujeres y el 40,6\% son varones. Los trabajadores provienen de instituciones y empresas de diversos rubros como de servicios educativos (tanto de nivel escolar como universitario) y servicios comerciales; otros son administrativos del sector público. La muestra fue seleccionada de manera no probabilística por medio de la técnica de grupos intactos.

\section{Instrumento}

La Escala de Satisfacción Familiar de Olson y Wilson consta de 14 items, de los cuales los impares valoran la dimensión de Cohesión y los pares la dimensión de Adaptabilidad. Este instrumento es una versión corta de la escala FACES II de Olson. Las alternativas de respuesta tienen cinco niveles en una escala tipo Likert. Los valores se interpretan en función del modelo circunflejo ${ }^{29}$ de modo que los valores extremos indicarían disfuncionalidad familiar y los valores medios, un equilibrio entre la cohesión y la adaptabilidad, que sería indicador de funcionalidad familiar. Validaciones previas hechas en Perú, para muestras de adolescentes, han reportado que presenta un índice de confiabilidad total de $\alpha=0,84$, y de $\alpha=0,76$ para la dimensión de Cohesión y de $\alpha=0,70$ para la de adaptabilidad. Asimismo, el análisis factorial exploratorio obtuvo un valor KMO de 0,902 y dos factores que explicaron el 51,92\% de la varianza total de la prueba. El análisis factorial confirmatorio reveló indicadores adecuados de bondad de ajuste de acuerdo con el modelo propuesto por Olson. ${ }^{24}$

\section{Procedimiento}

La recolección de los datos se llevó a cabo en los centros de trabajo de los participantes, previa autorización de los jefes y responsables competentes en cada caso. Se informó a los participantes acerca de los fines del estudio y firmaron un consentimiento informado en el protocolo de respuesta del instrumento utilizado para esta investigación. 


\section{REFERENCIAS}

1. Rubio C, Osca A, Recio P, Urien B, Peiró JM. Work-family conflict, self-efficacy, and emotional exhaustion: A test of longitudinal effects. J Work Organ Psychol. 2015; 31(3):147-154.

2. Quiroga MA, Sánchez MP. Análisis de la insatisfacción familiar. Psicothema. 1997; 9(1):69-92.

3. Quezada J, Zavala $E_{1}$ Lenti M. Satisfacción familiar en mujeres jóvenes. Av Psicol. 2015; 23(2): 223-229.

4. Pinto J, Barra E. Conflicto trabajo-familia y bienestar psicológico en trabajadores de empresas industriales de Chile. Rev Per Psic Trab Soc. 2015; 4(1):15-24.

5. Golik M. Las expectativas de equilibrio entre vida laboral y vida privada y las elecciones laborales de la nueva generación. Cuad Adm. 2013; 26(46): 107-133.

6. Cachutt $\mathrm{C}$, Ortiz F. Mediación de la integración trabajo-familia en las organizaciones. Ind Data. 2015; 18(2):7-13.

7. Arias WL, Castro R, Dominguez S, Masias MA, Canales F, Castilla S, Castilla S. Construcción de un inventario de integración familiar. Av Psicol. 2013; 21(2): 195-206.

8. León F. Las psicologias del área social-organizacional en el Perú: 20032012. Rev Psicol (Lima). 2013; 31(2):177-226.

9. Prado TR, Del Águila M. Ajuste y satisfacción en parejas que trabajan. Rev Invest Psicol-UNFV (Lima). 2010; 1: 38-52.

10. Arias WL, Ceballos K. Síndrome de burnout, satisfacción laboral e integración familiar en trabajadores de una tienda por departamento de Arequipa. Illustro. 2016; 7:43-58.

11. Rivas AA. Tipos de sistema familiar y riesgo suicida en adolescentes. Rev Psicol (Arequipa). 2008; 5:31-43.

12. Laguna JP, Rodríguez AS. Comportamientos socioemocionales de resiliencia en preescolares procedentes de hogares mono y biparentales. Rev Psicol (Arequipa). 2008; 5:52-65.

13. Arias $W L$, Quispe $A C$, Ceballos $K D$. Estructura familiar y nivel de logro en niños y niñas de escuelas públicas de Arequipa. Perspect fam. 2016; 1:35-62.

14. Delgado P. Estrategias de negociación en parejas violentas y no violentas de Arequipa. Perspect fam. 2016; 1:11-22.

15. Silva $C$, Argote $C$. Actitudes hacia matrimonio y divorcio en jóvenes procedentes de familias intactas y divididas. Rev Psicol (Arequipa). 2007; 4: 29-37.

16. Murillo MG. Desarrollo del juicio moral en personas casadas y divorciadas en la ciudad de Arequipa. Av Psicol. 2015; 23(1):73-85.

17. Seperak R. Motivos extrínsecos-intrínsecos y trascendentes en la decisión de contraer matrimonio en casados, separados y/o divorciados. Rev Psicol (Arequipa). 2016; 6(1):349-368.

18. Arias $W L$, Masias MA, Salas $X S$, Yepez $L$, Justo 0 . Integración familiar y felicidad en la ciudad de Arequipa. Rev Psicol (Arequipa). 2014; 4(2):204215.

19. Barreda A, Bouroncle $S$, Corrales AM, Torres L. La mujer en la dirección desde la visión de género. Rev Psicol (Arequipa). 2014; 4(1):67-79.

20. Riesco R, Arela R. Impacto de la estructura familiar en la satisfacción con los ingresos en los hogares urbanos en Perú. Economía; 2015; 38(76):51-76.

21. Castro RJ, Riesco G, Arela R. ¿Familia y bienestar? Explorando la relación entre estructura familiar y satisfacción con la vida personal de las familias. Bol-Acad Paul Psicol. 2016; 36(90):86-104.
22. Castro $R$, Arias $W L$, Dominguez $S$, Masias $M A$, Salas $X$, Canales $F$, Flores $A$. Integración familiar y variables socioeconómicas en Arequipa metropolitana. Rev Investig (Arequipa). 2013; 4:35-65.

23. Castilla $H$, Caycho $T$, Shimabukuro $M$, Valdivia A. Percepción del funcionamiento familiar: Análisis psicométrico de la Escala APGAR-familiar en adolescentes de Lima. Propósitos y Representaciones; 2014; 2(1):49-63.

24. Capa W, Vallejos $M$, Cárdenas R. Factores psicosociales asociados al consumo de drogas en adolescentes de una zona urbano popular de Lima Metropolitana. Rev Invest Psicol-UNFV (Lima). 2010; 1:21-37.

25. Mayorga $E_{1}$ Ñiquen M. Satisfacción familiar y expresión de la cólerahostilidad en adolescentes escolares que presentan conductas antisociales. Rev Invest Psicol-UNFV (Lima). 2010; 1:87-92.

26. Bazo-Alvarez JC, Bazo-Alvarez OA, Aguila J, Peralta F, Mormontoy W, Benett IM. Propiedades psicométricas de la Escala de Funcionalidad Familiar FACES-III: Un estudio en adolescentes peruanos. Rev Perú Med Exp Salud Publica. 2016; 33(3):462-470.

27. Martínez-Pérez MD, Osca A. Análisis psicométrico del Inventario de Apoyo Familiar para trabajadores. Psichotema. 2002; 14(2):310-316.

28. Olson DH. Circumplex model VII: validation studies and FACES III. Fam Process. 1986; 25(3): 337-351.

29. Olson DH, Russell C, Sprenkle DH. Circumplex model of marital and family systems II: Empirical studies and clinical intervention. Adv Fam Interv Asses Theory. 1989; 1:129-176.

30. Olson DH. Circumplex model of marital and family systems. J Fam Ther. 2000; 22(2):144-67.

31. Schmidt V, Barreyro JP, Maglio AL. Escala de evaluación del funcionamiento familiar FACES III: ¿Modelo de dos o tres factores?. Escritos de Psicología. 2009; 3(2):30-36.

32. Reusche R. M. Estructura y funcionamiento familiar en un grupo de estudiantes de secundaria de nivel socioeconómico medio con alto y bajo rendimiento escolar. Rev Psicol-UNIFE (Lima). 1995; 3:163-190.

33. Ferreira AM. Sistema de interacción familiar asociado a la autoestima de menores de situación de abandono moral o prostitución. Rev Invest PsicolUNFV (Lima). 2003; 6(2):58-80.

34. Montero I, León OG. A guide for naming research studies in Psychology. Int J Clin HIth Psyc. 2007; 7(3):847-862.

35. IBM. SPSS Statistics (Version 20) [software CD-ROM]. Nueva York: IBM; 2011.

36. Arbuckle J. IBM SPSS Amos 21 User's Guide. New York: IBM; 2013.

37. Jöreskog K. A general approach to confirmatory maximun likelihood factor analysis. Psychometrika. 1969; 34:183-202.

38. Byrne B. Structural equation modeling with AMOS. 2a ed. New York: Routledge, Taylor \& Francis Group; 2010.

39. Arias WL, Ceballos KD, Román A, Maquera C, Sota A. Impacto de la familia en el trabajo: un estudio predictivo en trabajadores de una universidad privada de Arequipa. Rev Psicol (Arequipa). (no publicado); 2016.

40. Underhill AT, LoBello SG, Fine PR. Reliability and validity of the Familiy Satisfaction Scale with survivors of traumatic brain injury. J Rehabil Res Dev. 2004; 41(4):603-610.

41. Vega A, Moyano E. Adicción al trabajo, satisfacción laboral y familiar en académicos de una universidad estatal chilena. Salud \& Sociedad. 2010; 1(3):222-232 
Tabla 4.

Baremos de la Escala de Satisfacción familiar.

\begin{tabular}{|c|c|c|c|c|}
\hline & & Cohesión & Adaptabilidad & $\begin{array}{l}\text { Satisfacción } \\
\text { familiar }\end{array}$ \\
\hline Media & & 25,96 & 25,57 & 51,53 \\
\hline Desviación & típica & 5,183 & 5,052 & 9,946 \\
\hline Mínimo & & 7 & 7 & 14 \\
\hline Máximo & & 35 & 35 & 70 \\
\hline Alpha de C & ronbach & 0,858 & 0,848 & - \\
\hline & Percentiles & & ntuaciones direct & \\
\hline Baja & 5 & 17,00 & 17,00 & 34,00 \\
\hline & 10 & 20,00 & 20,00 & 40,00 \\
\hline & 15 & 21,00 & 21,00 & 42,00 \\
\hline & 20 & 22,00 & 21,00 & 44,00 \\
\hline & 25 & 23,00 & 22,00 & 45,00 \\
\hline Promedio & 30 & 23,50 & 23,00 & 46,00 \\
\hline & 35 & 24,00 & 24,00 & 48,00 \\
\hline & 40 & 25,00 & 24,00 & 49,00 \\
\hline & 45 & 25,00 & 25,00 & 50,75 \\
\hline & 50 & 26,00 & 26,00 & 52,00 \\
\hline & 55 & 27,00 & 27,00 & 54,00 \\
\hline & 60 & 28,00 & 27,00 & 55,00 \\
\hline & 65 & 28,00 & 28,00 & 56,00 \\
\hline & 70 & 29,00 & 28,00 & 56,00 \\
\hline Alta & 75 & 29,00 & 28,00 & 58,00 \\
\hline & 80 & 30,00 & 30,00 & 60,00 \\
\hline & 85 & 31,00 & 31,00 & 62,00 \\
\hline & 90 & 33,00 & 32,00 & 64,00 \\
\hline & 95 & 35,00 & 35,00 & 68,50 \\
\hline
\end{tabular}

Los valores Chi-cuadrado de bondad de ajuste $\left(X^{2}=121,951 ; \mathrm{gl}=\right.$ $73 ; \mathrm{p}<0,001)$ nos indican que debemos rechazar el modelo; pero debido a que el valor $X^{2}$ tiende a llevarnos a rechazar los modelos propuestos cuando se trabaja con muestras grandes de sujetos ${ }^{37}$, las conclusiones se sustentaron en otros criterios como la razón entre la $X^{2}$ de bondad de ajuste sobre sus grados de libertad que es menor de $3(\mathrm{gl}=1,671)$. Además del índice de bondad de ajuste (GFI $=0,938)$, el índice de ajuste comparativo (CFI $=0,975)$, que tienen valores para las diversas subescalas mayores de 0,90. Asimismo, la raíz cuadrada de la media de los residuos $(\mathrm{RMR}=$ 0,0375) y la raíz cuadrada de media del error de aproximación (RMSEA $=0,050)$ son menores de 0,08. En general, estos índices son aceptables38 y nos refieren que los modelos tienen un buen ajuste, lo que indica que el instrumento tiene una estructura factorial válida.

Por otro lado, se evaluó la confiabilidad de los dos factores de la escala mediante el método de consistencia interna, haciendo uso del coeficiente Alpha de Cronbach. Como puede observarse en la Tabla 4, ambos factores muestran una adecuada confiabilidad con coeficientes mayores a 0,8. Además, se establecieron los baremos con los respectivos percentiles para los valores obtenidos. Estos baremos generaron tres rangos de respuesta, tanto para los factores de Cohesión y Adaptabilidad así como para la escala de satisfacción familiar en general. Estos baremos permiten ubicar en los niveles bajo, promedio y alto los puntajes obtenidos por los trabajadores evaluados, de manera que se puede ofrecer un diagnóstico psicométrico con la prueba, ya sea con fines de investiga- ción o de valoración de la satisfacción familiar siguiendo los lineamientos teóricos del modelo circunflejo.

\section{DISCUSIÓN}

La Escala de Satisfacción Familiar de Olson ha sido aplicada extensamente para valorar diversas cuestiones sobre el funcionamiento familiar, en niños ${ }^{33}$, en adolescentes ${ }^{25}$, en adultos ${ }^{39}$, y hasta en pacientes con lesión cerebral ${ }^{39} \mathrm{y}$ cuenta con diversos estudios con respecto a su validación y sus propiedades psicométricas, que sobrepasan largamente el millar de estudios. ${ }^{31}$ Sin embargo, en los países de la región no existen investigaciones psicométricas sobre dicho instrumento en muestras de trabajadores, muy a pesar de que en la actualidad es fundamental la valoración de la satisfacción y la funcionabilidad familiar como un factor potencial de riesgo psicosocial para el trabajador.

Aunque es posible encontrar diversos estudios en América Latina sobre el conflicto trabajo-familia ${ }^{4,6,9,10,39,41}$, no se cuenta con instrumentos validados para estos fines, de manera específica, ni con respecto a la variable familia. Por ello, la principal motivación de este trabajo fue el estudio psicométrico de la Escala de Satisfacción Familiar, que implicó su aplicación a 274 trabajadores de la ciudad de Arequipa. Los resultados indican que la escala cuenta con un buen nivel de confiabilidad, así como de validez de constructo. El análisis factorial exploratorio practicado no permitió replicar la saturación de los items en las dimensiones de Cohesión y Adaptabilidad, como originalmente se ha propuesto ${ }^{29,24}$, sin embargo, el análisis factorial confirmatorio reprodujo con adecuados índices de bondad de ajuste la estructura bifactorial de la escala. Además, como producto de nuestro análisis se han derivado los baremos necesarios para valorar el grado de cohesión y adaptabilidad de los trabajadores, de acuerdo a la funcionalidad familiar.

En ese sentido, una limitación de este estudio es que sus resultados y los baremos son aplicables a muestras de trabajadores que residen en la ciudad de Arequipa. Por ello, es importante validar este instrumento en diversos contextos organizacionales de Latinoamérica, además de que sería conveniente aumentar la cantidad de participantes, para conseguir resultados más fiables con muestras representativas. A pesar de ello, el presente estudio pone énfasis en la necesidad de valorar el contexto familiar de los trabajadores con instrumentos apropiados para la realidad concreta en que se desenvuelven. A futuro es importante que en América Latina se siga profundizando más sobre el tema del conflicto familia-trabajo, que en los países de Europa constituye un asunto de vital importancia, dadas las características sociodemográficas de sus países. ${ }^{1}$ Dado que en los países de la región tendemos a reproducir los modos de vida occidentales, sería conveniente investigar el impacto de los asuntos familiares en el trabajo y viceversa, para promover un balance adecuado entre estos dos escenarios, que ocupan la mayor parte del tiempo de las personas durante su vida adulta. Es fundamental que tanto la familia como el trabajo sean espacios para el despliegue de las potencialidades humanas y vehículos sociales del bienestar psicológico. 
Tabla 1.

Estadísticos descriptivos.

\begin{tabular}{lccccccccc}
\multicolumn{1}{c}{ N } & Mín. & Máx. Media & D.E. & Asimetría & ET & Curtosis & ET \\
İtem 1 & 274 & 1 & 5 & 3,92 & 1,002 & $-1,113$ & 0,147 & 1,152 & 0,293 \\
İtem 2 & 274 & 1 & 5 & 3,81 & 0,992 & $-0,850$ & 0,147 & 0,522 & 0,293 \\
İtem 3 & 274 & 1 & 5 & 3,61 & 1,000 & $-0,526$ & 0,147 & 0,032 & 0,293 \\
İtem 4 & 274 & 1 & 5 & 3,31 & 1,100 & $-0,249$ & 0,147 & $-0,451$ & 0,293 \\
İtem 5 & 274 & 1 & 5 & 3,57 & 1,125 & $-0,609$ & 0,147 & $-0,176$ & 0,293 \\
İtem 6 & 274 & 1 & 5 & 3,61 & 1,011 & $-0,398$ & 0,147 & $-0,267$ & 0,293 \\
İtem 7 & 274 & 1 & 5 & 3,55 & 0,979 & $-0,297$ & 0,147 & $-0,356$ & 0,293 \\
İtem 8 & 274 & 1 & 5 & 3,71 & 0,981 & $-0,560$ & 0,147 & 0,100 & 0,293 \\
İtem 9 & 274 & 1 & 5 & 3,72 & 1,004 & $-0,549$ & 0,147 & $-0,136$ & 0,293 \\
İtem 10 & 274 & 1 & 5 & 3,64 & 0,986 & $-0,495$ & 0,147 & $-0,039$ & 0,293 \\
Ittem 11 & 274 & 1 & 5 & 3,81 & 0,941 & $-0,658$ & 0,147 & 0,326 & 0,293 \\
İtem 12 & 274 & 1 & 5 & 3,80 & 0,960 & $-0,774$ & 0,147 & 0,451 & 0,293 \\
İtem 13 & 274 & 1 & 5 & 3,78 & 0,995 & $-0,568$ & 0,147 & $-0,220$ & 0,293 \\
İtem 14 & 274 & 1 & 5 & 3,69 & 0,946 & $-0,530$ & 0,147 & 0,189 & 0,293
\end{tabular}

Tabla 2.

Varianza total explicada.

\begin{tabular}{|c|c|c|c|c|c|c|c|}
\hline \multirow[t]{2}{*}{ Factor } & \multicolumn{3}{|c|}{$\begin{array}{c}\text { Autovalores } \\
\text { iniciales }\end{array}$} & \multicolumn{3}{|c|}{$\begin{array}{c}\text { Sumas de las } \\
\text { saturaciones al cuadrado } \\
\text { de la extracción }\end{array}$} & \multirow{2}{*}{$\begin{array}{c}\text { Suma de las } \\
\text { saturaciones } \\
\text { al cuadrado } \\
\text { de la rotación } \\
\text { Total }\end{array}$} \\
\hline & Total & $\begin{array}{c}\% \text { de la } \\
\text { varianza }\end{array}$ & $\begin{array}{c}\% \\
\text { acumulado }\end{array}$ & Total & $\begin{array}{c}\% \text { de la } \\
\text { varianza }\end{array}$ & $\begin{array}{c}\% \\
\text { acumulado }\end{array}$ & \\
\hline 1 & 7,151 & 51,078 & 51,078 & 6,631 & 47,366 & 47,366 & 6,322 \\
\hline 2 & 1,075 & 7,680 & 58,758 & 0,677 & 4,839 & 52,205 & 5,422 \\
\hline 3 & 0,755 & 5,391 & 64,148 & & & & \\
\hline 4 & 0,716 & 5,115 & 69,264 & & & & \\
\hline 5 & 0,656 & 4,685 & 73,949 & & & & \\
\hline 6 & 0,563 & 4,020 & 77,968 & & & & \\
\hline 7 & 0,526 & 3,756 & 81,725 & & & & \\
\hline 8 & 0,463 & 3,307 & 85,032 & & & & \\
\hline 9 & 0,436 & 3,113 & 88,144 & & & & \\
\hline 10 & 0,401 & 2,862 & 91,006 & & & & \\
\hline 11 & 0,365 & 2,605 & 93,611 & & & & \\
\hline 12 & 0,353 & 2,521 & 96,132 & & & & \\
\hline 13 & 0,319 & 2,280 & 98,412 & & & & \\
\hline 14 & 0,222 & 1,588 & 100,000 & & & & \\
\hline
\end{tabular}

\section{Análisis de datos}

El análisis estadístico se realizó mediante el software SPSS 20.035 para el cálculo de la confiabilidad; además del módulo SPSS AMOS ${ }^{36}$ para el análisis factorial confirmatorio (AFC).

\section{RESULTADOS}

En primer término, se tienen los estadísticos descriptivos en la Tabla 1 , donde se aprecian los puntajes promedio por ítem con respecto a los valores mínimos y máximos y la desviación estándar. También se tiene la asimetría y la curtosis con su respectivo error típico. De todo ello se observa que los puntajes tienden a ser moderados con tendencia a ubicarse por encima de la media.

Para verificar la estructura de la prueba, y determinar su validez de constructo, lo primero que se hizo fue valorar la adecuación muestral para ver si es posible aplicar un análisis factorial. El valor de la prueba Kaiser Meyer Olkin (KMO) fue de 0,940 y la prueba de esfericidad de Bartlett arrojó un valor Chi-cuadrado de 1986.485 con 91 grados de libertad y altamente significativo $(\mathrm{p}<$ $0,000)$; por lo que se concluye que los datos pueden someterse a un análisis factorial. Seguidamente entonces, realizó la factoriza- ción por medio del método de máxima verosimilitud, encontrándose dos factores que explican el 52,205\% de la varianza total de la prueba: el primer factor explica el $47,366 \%$ de la varianza total y el segundo factor explica el 4,839\% de la varianza total (ver Tabla 2).

Se obtuvo asimismo una matriz de dos factores con las saturaciones de los ítems, mediante el método de rotación Promax con Kaiser. En el factor 1 saturaron los ítems 3, 4, 5, 6, 7, 8, 9, 10, 11, 13 y 14; mientras que en el factor 2 saturaron los ítems 1, 2 y 12 (ver Tabla 3). Asimismo, la correlación entre los dos factores resultantes fue de 0,749 mediante el coeficiente de correlación de Pearson, lo que sugiere que ambos factores se encuentran positiva y fuertemente correlacionados.

En segundo lugar, se realizó un análisis factorial confirmatorio (AFC) mediante la estimación por máxima verosimilitud. Utilizamos este tipo de análisis factorial teniendo en cuenta que la estructura bifactorial de la prueba se ha mantenido estable a lo largo de varios años de estudio, y particularmente en los estudios previos llevados a cabo en muestras peruanas. ${ }^{24-26,32}$

Tabla 3.

Matriz de estructura.

\begin{tabular}{lcc} 
& \multicolumn{3}{c}{ Factor } \\
Ítem 1 & 1 & 2 \\
İtem 2 & 0,584 & 0,820 \\
İtem 3 & 0,610 & 0,888 \\
İtem 4 & 0,585 \\
İtem 5 & 0,688 & 0,344 \\
İtem 6 & 0,501 & 0,420 \\
İtem 7 & 0,489 \\
İtem 8 & 0,599 & 0,535 \\
İtem 9 & 0,726 & 0,690 \\
İtem 10 & 0,702 & 0,578 \\
İtem 11 & 0,780 & 0,558 \\
İtem 12 & 0,697 & 0,626 \\
İtem 13 & 0,711 & 0,677 \\
İtem 14 & 0,721 & 0,698 \\
\hline
\end{tabular}

Figura 1.

AFC de la Escala de Satisfacción Familiar.

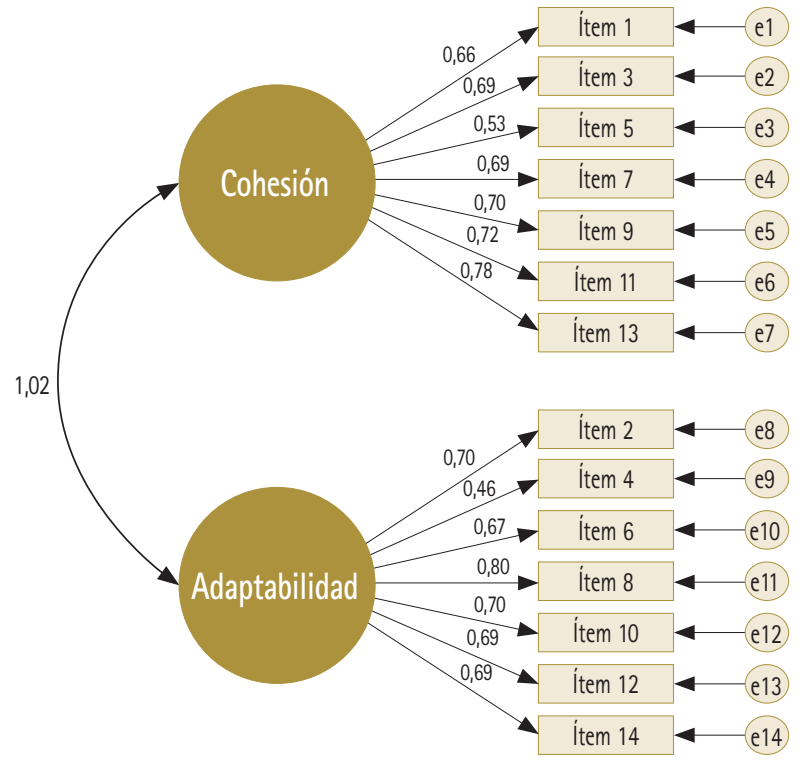

